\title{
Association of the Nonalcoholic Hepatic Steatosis and Its Degrees With the Values of Liver Enzymes and Homeostasis Model Assessment-Insulin Resistance Index
}

\author{
Mario Augusto Ferreira Cruz ${ }^{\mathrm{a}, \mathrm{c}}$, Josilda Ferreira Cruz ${ }^{\mathrm{a}}$, Larissa Baracho Macena ${ }^{\mathrm{a}}$, \\ Demetrius Silva de Santana ${ }^{\mathrm{b}}$, Cristiane Costa da Cunha Oliveira ${ }^{\mathrm{a}}$, Sonia Oliveira Lima ${ }^{\mathrm{a}}$, \\ Alex Vianey Callado Franca ${ }^{a}$
}

\begin{abstract}
Background: Nonalcoholic fatty liver disease (NAFLD) is among the most common chronic diseases of the modern world with a wide variety of factors including genetic, environmental and metabolic. The aim of this study was to verify the association between the degrees of hepatic steatosis at the abdominal ultrasound and the values of aminotransferases (aspartate aminotransferase (AST) and alanine transferase (ALT)), gamma glutamyl transpeptidase (GGT) and homeostasis model assessment-insulin resistance (HOMA-IR) index.
\end{abstract}

Methods: A prospective, descriptive survey study, using a quantitative analytical examination, was conducted from July 2013 to July 2014. In the statistical analysis, values were expressed as median, first and third quartiles. We used the nonparametric Kruskal-Wallis test to compare the medians between the degrees of steatosis, adopted a statistical significance of $5 \%(\mathrm{P} \leq 0.05)$ and used the statistical program SPSS 22.0.

Results: We diagnosed 233/800 (29.1\%) patients with hepatic steatosis on routine ultrasound, and $65.7 \%$ were female. Regarding degrees, 119 had grade $1(51.0 \%), 94$ grade $2(40.4 \%)$ and 20 grade 3 $(8.6 \%)$. The median age of the patients with grade 1,2 or 3 did not vary significantly $(\mathrm{P}>0.05)$. The median body mass index (BMI), although clinically important because of its elevation, did not differ significantly $(\mathrm{P}>0.05)$. ALT levels increased as the degree of hepatic steatosis has advanced as well as the levels of AST, GGT and HOMAIR. AST values showed a greater association with the severity of fatty liver $(\mathrm{P}=0.0001)$ than the ALT $(\mathrm{P}=0.001)$.

Conclusions: ALT, AST, GGT and HOMA-IR are associated to the degrees of hepatic steatosis on ultrasound and can help in the selection of patients for the liver histological evaluation.

Keywords: Fatty liver; Ultrasonography; Liver enzymes; Insulin re-

Manuscript accepted for publication September 24, 2015

${ }^{a}$ Department of Medicine, Tiradentes University, Aracaju 49032-490, Sergipe, Brazil

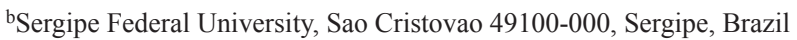

${ }^{\mathrm{c} C}$ Corresponding Author: Mario Augusto Ferreira Cruz, Department of Medicine, Tiradentes University, 300 Murilo Dantas Ave., Aracaju 49032-490, Sergipe, Brazil. Email: m.gutocruz@gmail.com

doi: http://dx.doi.org/10.14740/gr685w sistance

\section{Introduction}

Nonalcoholic fatty liver disease (NAFLD) covers the entire spectrum of fatty liver disease in individuals without significant consumption of alcohol, ranging from simple steatosis to steatohepatitis and liver cirrhosis [1]. NAFLD is present when the deposit of lipids, particularly triglycerides, exceeds $5 \%$ of total weight $[1,2]$.

NAFLD is among the most common chronic diseases of the modern world with a wide variety of factors including genetic, environmental and metabolic, with prevalence by ultrasonography (US) in industrialized countries ranging from $12.2 \%$ to $40 \%$ [3-9].

An important factor related to the emergence of nonalcoholic steatohepatitis (NASH) is the presence of insulin resistance (IR). This is defined as a reduced biological response to the actions of insulin, causing the fat, muscle and liver tissues to become unable to metabolize glucose and fatty acids, being exacerbated by obesity and the intake of dietary fats $[10,11]$. Thus, the association between IR and deposition of triglycerides in the liver, being evaluated by evaluation model index or the homeostasis model assessment-IR (HOMA-IR) is an important instrument [12]. The reference point for the diagnosis of IR by HOMA-IR is controversial, being considered by the Brazilian Society of Diabetes $>3.60$ [13].

NAFLD patients are usually asymptomatic, with incidental diagnostic imaging or by changing aminotransferases. Abdominal US is widely used as first-line method for the investigation of NAFLD and others affections of liver, as a simple method that does not use ionizing radiation, less costly and more affordable $[14,15]$.

When change occurs in aminotransferases, there is usually increased alanine transferase (ALT), while aspartate aminotransferase (AST) levels remain normal or slightly elevated values. However, approximately $80 \%$ of patients have normal ALT levels. The use of ALT for diagnosis is controversial to determine severity of NAFLD [2, 16, 17]. The gamma-glutamyl transpeptidase (GGT) may be a biochemical finding early found in this disease, but also not specific, given being influenced by various factors such as hepatobiliary disease, alco- 


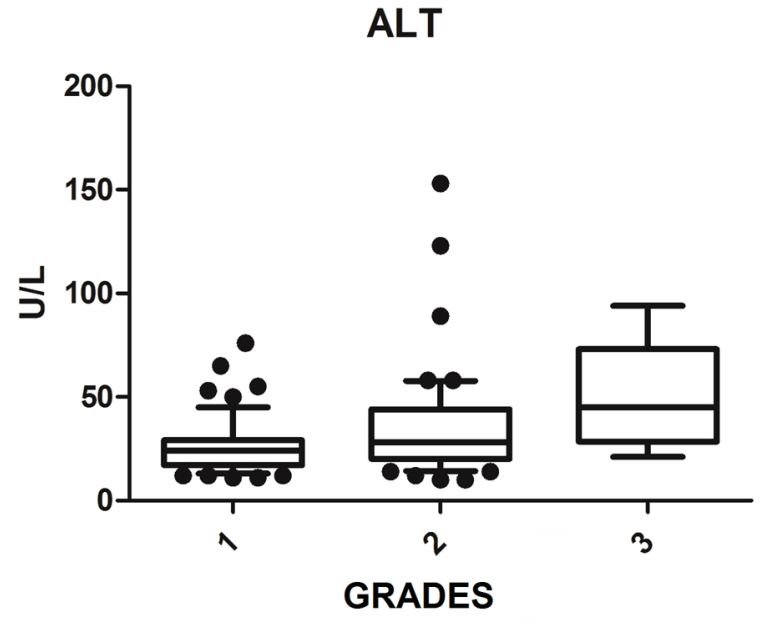

Figure 1. ALT vs. hepatic steatosis. ALT: alanine transferase; P: significance.

holism and drug use, making it extremely nonspecific [18]. It has been reported that GGT as a marker sensitive to IR, which may be an early biochemical finding of NAFLD [19].

The aim of this study was to investigate the association between the degrees of hepatic steatosis at abdominal ultrasound and amounts of aminotransferases (AST and ALT), GGT and HOMA-IR.

\section{Material and Methods}

A prospective, descriptive survey type, with analytical and quantitative approach, was conducted. We analyzed 800 patients who underwent the examination of US for various clinical conditions, excluding those with alcohol consumption $>40$ $\mathrm{g}$ /day and previous liver diseases, from July 2013 to July 2014, starting after approval by the Ethics and Research Committee with protocol $010513 \mathrm{R}$. We submitted patients to the US and biochemical analysis of aminotransferases, GGT, fasting glucose and basal insulin. We conducted the examination of US with convex transducer, dynamic (with formation of the continuous and automatic image) of $3.75 \mathrm{MHz}$ frequency, performed by the same examiner (JFC), experienced in the diagnosis of hepatic steatosis image. US of steatosis classified the degrees based on the criteria of Saadeh et al (2002) [20]. The degree 0 is the normal test, the grade 1 is characterized by the display of fine echoes of the hepatic parenchyma with normal viewing of the diaphragm and intrahepatics vessels, grade 2 is characterized by diffuse increase in the fine echoes with impaired view of intrahepatic vessels and diaphragm, and grade 3 is characterized with a significant increase in fine echoes and impaired or absent visualization of intrahepatic vessels. All patients signed the written informed consent (WIC).

The AST and ALT were performed by NADH method (no P-5-P), and the determination of GGT used tubes with separator gel and ABBOTT reagent with the dosage based on the principle that GGT catalyzes the transfer of the gamma gluta-

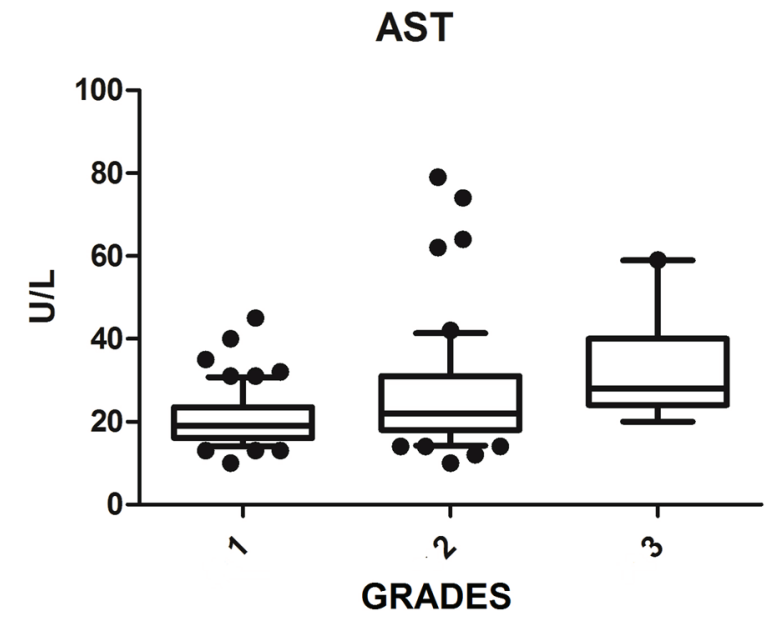

Figure 2. AST vs. hepatic steatosis. AST: aspartate aminotransferase; $P$ : significance.

myl group from the substrate 3-carboxy-4-nitroanilide glycylglycine receiver for producing 3-carboxy-4-nitroaniline. From the basal insulin to fasting glucose, we used the HOMA-IR index, using the form proposed by Matthews et al (1985) [12] (fasting insulin $\mathrm{U} / \mathrm{mL} \times$ fasting glucose, $\mathrm{mmol} / \mathrm{L} / 22.5$ ).

In the statistical analysis, values were expressed as median, first and third quartiles. We used the nonparametric Kruskal-Wallis test to compare the medians between the degrees of steatosis. We adopted a statistical significance of $5 \%$ $(\mathrm{P} \leq 0.05)$ and used the statistical program SPSS 22.0.

\section{Results}

We diagnosed 233/800 (29.1\%) patients by the US of hepatic steatosis, $153(65.7 \%)$ were female and $80(34.3 \%)$ were male. Regarding degrees, 119 had grade $1(51.0 \%), 94$ grade 2 $(40.4 \%)$ and 20 grade $3(8.6 \%)$. The median age of the patients with grade 1 was 44.0 years (first quartile and third quartile were 37.0 and 51.0), grade 2 was 47.5 years (40.0 and 53.0) and grade 3 was 45.5 (36.5 and 54.8), but did not vary significantly $(\mathrm{P}>0.05)$. The median body mass index (BMI), although clinically important because of its elevation, did not differ significantly $(P>0.05)$. ALT levels increased as the degree of advanced hepatic steatosis (Fig. 1) as well as the levels of AST, GGT and HOMA-IR, shown in Figures 2-4, respectively.

The median AST, ALT, GGT and HOMA-IR index showed statistically significant variation in the degree of hepatic steatosis $(\mathrm{P} \leq 0.05)$, as shown in Table 1 . The AST showed a greater association with the severity of steatosis $(\mathrm{P}=0.0001)$ than the ALT $(\mathrm{P}=0.001)$.

\section{Discussion}

The prevalence of alcoholic hepatic steatosis does not vary 


\section{GGT}

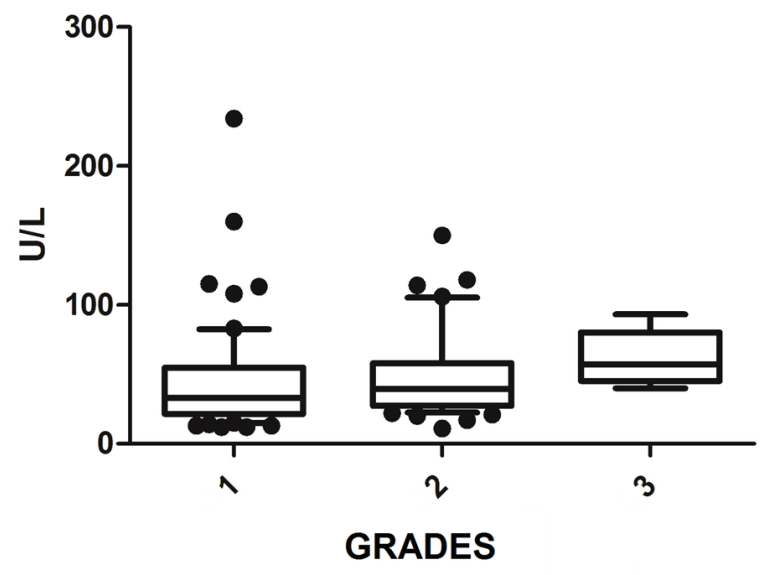

Figure 3. GGT vs. hepatic steatosis. GGT: gamma-glutamyl transpeptidase; $P$ : significance.

depending on the region studied and the diagnostic methods used. Eastern countries often have a lower prevalence compared to the West, as the Western lifestyle is, in itself, an important environmental risk factor. The prevalence of NASH by the US is $40 \%$ in Germany [3], 27.3\% in South Korea [4], 25.8\% in Spain [5], 20\% in Italy [6], 17.2\% in China [7] and $12.2 \%$ in the Philippines [8]. In Brazil, a previous study showed a prevalence of $23 \%$ [9]. This study showed that $29.1 \%$ of adults surveyed in the city of Aracaju, Sergipe, Brazil, had NAFLD. This disease of the liver has a high frequency in many countries, which was corroborated with the Brazilian present study, one of the most prevalent chronic diseases in the world today.

Currently ultrasound criteria are mainly used to evaluate the severity of hepatic steatosis, for example, the portal vein flow velocity and the hepatic artery resistance index, because current studies show that the portal vein flow velocity and the hepatic artery resistance index decrease with increasing severity [21, 22]..However, it is not a unanimity [23], and it has

\section{HOMA-IR}

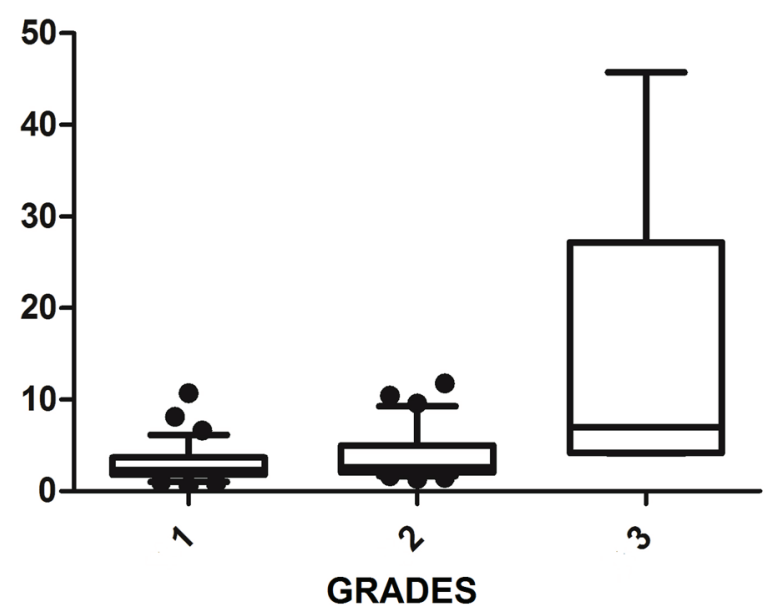

Figure 4. HOMA-IR vs. hepatic steatosis. HOMA-IR: homeostasis model assessment-insulin resistance; P: significance.

researched involving the liver and metabolic markers that may assist in this evaluation.

Elevated ALT levels appear to be associated with NAFLD clinically and histologically [24], since those levels reflect the eating habits of the patient, with decreasing levels observed after the consumption of a diet rich in vegetables and low in animal protein [25]. Bi et al (2014) evaluated more than 8,000 people and had higher values of AST and ALT in the group of patients with NAFLD compared to the control group, and found that patients with NAFLD with advanced degrees had significantly increased aminotransferase values, thus, there was strong correlation between the levels of these enzymes and NAFLD [26]. This fact was corroborated by Tomizawa et al (2014) that showed that AST and ALT were significantly higher in patients with NAFLD compared to those without NAFLD $(P=0.0001)$ [27] and also by this study, which found a significant association of AST and ALT levels with increased ultrasound degrees of hepatic steatosis. The aminotransferase can be used to predict the degree of NAFLD.

Table 1. Variables Medians vs. Severity of Hepatic Steatosis

\begin{tabular}{|c|c|c|c|c|c|c|c|}
\hline & \multicolumn{6}{|c|}{ Nonalcoholic hepatic steatosis } & \multirow{3}{*}{$\mathbf{P}$} \\
\hline & \multicolumn{2}{|l|}{ Grade 1} & \multicolumn{2}{|l|}{ Grade 2} & \multicolumn{2}{|l|}{ Grade 3} & \\
\hline & Median (quartile) & $\mathbf{n}$ & Median (quartile) & $\mathbf{n}$ & Median (quartile) & $\mathbf{n}$ & \\
\hline Age $(n=233)$ & $44.0(37.0-51.0)$ & 119 & $47.5(40.0-53.0)$ & 94 & $45.5(36.5-54.8)$ & 20 & 0.107 \\
\hline $\operatorname{BMI}(\mathrm{n}=125)$ & $29.5(26.5-32.4)$ & 64 & $30.4(27.9-35.2)$ & 54 & $33.6(29.2-45.2)$ & 7 & 0.129 \\
\hline GGT $(\mathrm{n}=127)$ & $34.0(21.5-55.5)$ & 65 & $39.5(28.5-62.0)$ & 56 & $57.0(42.2-80.2)$ & 6 & 0.034 \\
\hline $\operatorname{AST}(n=135)$ & $19.0(16.0-23.0)$ & 68 & $23.0(18.0-31.2)$ & 58 & $28.0(24.0-40.0)$ & 9 & 0.0001 \\
\hline $\operatorname{ALT}(\mathrm{n}=136)$ & $24.0(17.0-28.5)$ & 69 & $29.5(20.7-44.0)$ & 58 & $45.0(28.5-73.0)$ & 9 & 0.001 \\
\hline HOMA-IR $(\mathrm{n}=75)$ & $2.34(1.88-3.58)$ & 36 & $2.62(2.04-5.23)$ & 34 & $6.98(4.2-27.2)$ & 5 & 0.008 \\
\hline
\end{tabular}

BMI: body mass index; GGT: gamma-glutamyl transpeptidase; AST: aspartate aminotransferase; ALT: alanine transferase; HOMA-IR: homeostasis model assessment-insulin resistance; $n$ : number of patients; P: significance. 
However, there is controversy in the literature about the use of aminotransferases in assessing the severity of NAFLD. McPherson et al (2010) and Dyson et al (2014) showed in their studies that the ALT values do not correlate with histological findings and are of little use to determine the severity [2, 17]. The progression to more advanced levels can be noted in several patients with normal ALT, and in addition, the increase in ALT not associated with hepatic steatosis is observed in obese individuals, which impairs their use in clinical practice [28, 29].

The increase in GGT can be found early in NAFLD [18]; however, Bi et al (2014) showed that ALT is a more specific marker than GGT [26]. In the Portuguese population sample, Martins et al (2010) investigated the association of serum GGT to each of the risk factors for metabolic syndrome, being particularly strong with IR and being related to NAFLD [30]. This study found that whenever NAFLD hypothesis is clinically put, GGT values should be obtained, since it is a simple and important marked IR and cardiovascular risk factor [27]. This study found a statistically significant association between increased GGT and degrees of NASH, and may be useful for evaluation and monitoring of patients with NAFLD.

The combination of US of the liver and HOMA-IR is a sensitive and specific method not only to diagnose steatosis, but also in the prediction of severity [31, 32]. Damiani et al (2011) have also shown a strong relationship between IR measured by HOMA-IR and hepatic fat. Fedchuk et al (2014) found a median HOMA-IR 3.3 (2.3 - 5.6) between patients with NAFLD [33]. Moreover, the risk of IR increased 2.33fold in patients with NAFLD, and coexistence of NAFLD and elevated ALT were significantly associated with increased HOMA-IR amounting to a 4.65 times higher risk of IR. Thus, the study found that the coexistence of NAFLD and elevated ALT are associated with IR and can be useful for early detection of IR [34]. This study found that the HOMA-IR index is statistically associated with the evolution of the degrees of hepatic steatosis with a median of 6.98 (4.2 - 27.2) among patients with liver disease on most ultrasound graduation. Thus, the HOMA-IR index is an important tool that assists in the investigation of NAFLD, and can predict progression to more advanced degrees.

\section{Conclusion}

ALT, AST, GGT and HOMA-IR are related to the degrees of hepatic steatosis on ultrasound, and can help in the selection of patients for liver histological evaluation.

\section{Financial Support}

None.

\section{Competing Interests}

None of the authors have any competing interests.

\section{References}

1. Chalasani N, Younossi Z, Lavine JE, Diehl AM, Brunt EM, Cusi K, Charlton M, et al. The diagnosis and management of non-alcoholic fatty liver disease: practice Guideline by the American Association for the Study of Liver Diseases, American College of Gastroenterology, and the American Gastroenterological Association. Hepatology. 2012;55(6):2005-2023.

2. Dyson JK, Anstee QM, McPherson S. Non-alcoholic fatty liver disease: a practical approach to diagnosis and staging. Frontline Gastroenterol. 2014;5(3):211-218.

3. Kirovski G, Schacherer D, Wobser H, Huber H, Niessen C, Beer C, Scholmerich J, et al. Prevalence of ultrasounddiagnosed non-alcoholic fatty liver disease in a hospital cohort and its association with anthropometric, biochemical and sonographic characteristics. Int J Clin Exp Med. 2010;3(3):202-210.

4. Jeong EH, Jun DW, Cho YK, Choe YG, Ryu S, Lee SM, Jang EC. Regional prevalence of non-alcoholic fatty liver disease in Seoul and Gyeonggi-do, Korea. Clin Mol Hepatol. 2013;19(3):266-272.

5. Caballeria L, Pera G, Auladell MA, Toran P, Munoz L, Miranda D, Aluma A, et al. Prevalence and factors associated with the presence of nonalcoholic fatty liver disease in an adult population in Spain. Eur J Gastroenterol Hepatol. 2010;22(1):24-32.

6. Bedogni G, Miglioli L, Masutti F, Tiribelli C, Marchesini G, Bellentani S. Prevalence of and risk factors for nonalcoholic fatty liver disease: the Dionysos nutrition and liver study. Hepatology. 2005;42(1):44-52.

7. Li H, Wang YJ, Tan K, Zeng L, Liu L, Liu FJ, Zhou TY, et al. Prevalence and risk factors of fatty liver disease in Chengdu, Southwest China. Hepatobiliary Pancreat Dis Int. 2009;8(4):377-382.

8. De Lusong MA, Labio E, Daez L, Gloria V. Non-alcoholic fatty liver disease in the Philippines: comparable with other nations? World J Gastroenterol. 2008;14(6):913917.

9. Rocha R, Cotrim HP, Bitencourt AG, Barbosa DB, Santos AS, Almeida Ade M, Cunha B, et al. Nonalcoholic fatty liver disease in asymptomatic Brazilian adolescents. World J Gastroenterol. 2009;15(4):473-477.

10. Pimentel GD, Moreto F, Corrente JE, Portero-McLellan $\mathrm{KC}$, Burini RC. [Relationship of pattern hyperlipidic intake with quality of diet, insulin resistance and homocysteinemia in adults]. Acta Med Port. 2011;24(5):719-726.

11. Faria ER, Faria FR, Franceschini Sdo C, Peluzio Mdo C, Sant Ana LF, Novaes JF, Ribeiro SM, et al. [Insulin resistance and components of metabolic syndrome, analysis by gender and stage of adolescence]. Arq Bras Endocrinol Metabol. 2014;58(6):610-618.

12. Matthews DR, Hosker JP, Rudenski AS, Naylor BA, Treacher DF, Turner RC. Homeostasis model assessment: insulin resistance and beta-cell function from fasting plasma glucose and insulin concentrations in man. Diabetologia. 1985;28(7):412-419.

13. Sociedade Brasileira de Diabetes [organizacao Jose Egid- 
io Paulo de Oliveira,Sergio Vencio]. Diretrizes da sociedade brasileira de diabetes: 2013-2014. Sao Paulo: AC Farmaceutica, 2014.

14. Boente LA, Cootrim HP, Matteoni LA, Leal RA, Campo FD, Soares D. Importancia da ultrassonografia no diagn stico da doenca hepatica gordurosa nao alcoolica. Gastroenterologia Endoscopia Digestiva (GED). 2007;26(1):S2.

15. Assya N, Assya N, Samuela N, Lermanc A, Nsei W. Approach to Solid Liver Masses in the Cirrhotic Patient. Gastroenterology Research, 2009;2(5):259-267.

16. Mofrad P, Contos MJ, Haque M, Sargeant C, Fisher RA, Luketic VA, Sterling RK, et al. Clinical and histologic spectrum of nonalcoholic fatty liver disease associated with normal ALT values. Hepatology. 2003;37(6):12861292.

17. McPherson S, Stewart SF, Henderson E, Burt AD, Day CP. Simple non-invasive fibrosis scoring systems can reliably exclude advanced fibrosis in patients with nonalcoholic fatty liver disease. Gut. 2010;59(9):1265-1269.

18. Salgado AL, Carvalho L, Oliveira AC, Santos VN, Vieira $\mathrm{JG}$, Parise ER. Insulin resistance index (HOMA-IR) in the differentiation of patients with non-alcoholic fatty liver disease and healthy individuals. Arq Gastroenterol. 2010;47(2):165-169.

19. Adams LA, Lindor KD. Nonalcoholic fatty liver disease. Ann Epidemiol. 2007;17(11):863-869.

20. Saadeh S, Younossi ZM, Remer EM, Gramlich T, Ong JP, Hurley M, Mullen KD, et al. The utility of radiological imaging in nonalcoholic fatty liver disease. Gastroenterology. 2002;123(3):745-750.

21. Mohammadinia AR, Bakhtavar K, Ebrahimi-Daryani N, Habibollahi P, Keramati MR, Fereshtehnejad SM, Abdollahzade S. Correlation of hepatic vein Doppler waveform and hepatic artery resistance index with the severity of nonalcoholic fatty liver disease. J Clin Ultrasound. 2010;38(7):346-352.

22. Topal NB, Orcan S, Sigirli D, Orcan G, Eritmen U. Effects of fat accumulation in the liver on hemodynamic variables assessed by Doppler ultrasonography. J Clin Ultrasound. 2015;43(1):26-33.

23. Shateria K, Mohammadib A, Moloudic F, Nosaird E, Ghasemi-Rade M. Correlation Between Sonographic Portal Vein Diameter and Flow Velocity With the Clinical Scoring Systems MELD and CTP in Cirrhotic Patients: Is There a Relationship? Gastroenterology Research. 2012;5(3):112-119.

24. Neuschwander-Tetri BA, Clark JM, Bass NM, Van Natta
ML, Unalp-Arida A, Tonascia J, Zein CO, et al. Clinical, laboratory and histological associations in adults with nonalcoholic fatty liver disease. Hepatology. 2010;52(3):913-924.

25. Iwamoto M, Yagi K, Yazumi K, Komine A, Shirouchi B, Sato M. Eating a healthy lunch improves serum alanine aminotransferase activity. Lipids Health Dis. 2013;12:134.

26. Bi WR, Yang CQ, Shi Q, Xu Y, Cao CP, Ling J, Wang XY. Large-scale analysis of factors influencing nonalcoholic fatty liver disease and its relationship with liver enzymes. Genet Mol Res. 2014;13(3):5880-5891.

27. Tomizawa M, Kawanabe Y, Shinozaki F, Sato S, Motoyoshi Y, Sugiyama T, Yamamoto S, et al. Triglyceride is strongly associated with nonalcoholic fatty liver disease among markers of hyperlipidemia and diabetes. Biomed Rep. 2014;2(5):633-636.

28. Clark JM, Brancati FL, Diehl AM. The prevalence and etiology of elevated aminotransferase levels in the United States. Am J Gastroenterol. 2003;98(5):960-967.

29. Kunde SS, Lazenby AJ, Clements RH, Abrams GA. Spectrum of NAFLD and diagnostic implications of the proposed new normal range for serum ALT in obese women. Hepatology. 2005;42(3):650-656.

30. Martins MC, Faleiro LL, Afonso B, Fonseca A. [Association of gamma glutamyltransferase, metabolic syndrome and cardiovascular risk]. Acta Med Port. 2010;23(4):579588.

31. Hegazy MA, Abdel-Rahmana HM, El-Gayarb DG, Aminc YH. Liver ultrasound is more sensitive in assessing the severity of nonalcoholic fatty liver disease with homeostasis model assessment-insulin resistance. Egypt Liver J. 2012;2:41-46.

32. Damiani D, Kuba VM, Cominato L, Dichtchekenian V, Menezes Filho HC. [Metabolic syndrome in children and adolescents: doubts about terminology but not about cardiometabolic risks]. Arq Bras Endocrinol Metabol. 2011;55(8):576-582.

33. Fedchuk L, Nascimbeni F, Pais R, Charlotte F, Housset C, Ratziu V. Performance and limitations of steatosis biomarkers in patients with nonalcoholic fatty liver disease. Aliment Pharmacol Ther. 2014;40(10):1209-1222.

34. Wang R, Lu Q, Feng J, Yin F, Qin C, Liu B, Liu Y, et al. Coexistence of non-alcoholic fatty liver disease with elevated alanine aminotransferase is associated with insulin resistance in young Han males. Endocrine. 2012;41(1):7075. 